\title{
ON COMPACTNESS AND SEQUENTIAL COMPACTNESS
}

\author{
NORMAN LEVINE
}

Abstract. Let $X$ be a compact space of cardinality less than or equal to $\aleph_{1}$. It is shown that $X$ is sequentially compact.

The classical example of a compact space which is not sequentially compact is $X\left\{X_{\alpha}: \alpha \in \Delta\right\}$ where $X_{\alpha}$ and $\Delta$ are each the unit interval.

Some of my colleagues raised the question: Is there a space of cardinality less than or equal to $c$ which is compact but not sequentially compact?

The purpose of this note is to answer this question in the negative, using the continuum hypothesis.

To this end we need the following

Definition. Let $\left\{x_{n}\right\}$ and $\left\{y_{n}\right\}$ be two infinite sequences in a set $X$. We will say that $\left\{x_{n}\right\}$ is eventually a subsequence of $\left\{y_{n}\right\}$ (and write $\left\{x_{n}\right\}$ $\left.\leqslant\left\{y_{n}\right\}\right)$ iff there exist integers $M$ and $n_{1}<n_{2}<\cdots$ such that $x_{M+i}=y_{n_{i}}$ for all $i \geqslant 1$.

It is clear that $\leqslant$ is transitive.

Lemma. Let $X$ be a set and for each $i \geqslant 1$, let $\mathbf{x}_{i}$ be an infinite sequence in $X$. Suppose further that for each $i$ and $j$, either $\mathbf{x}_{i} \mathbf{x}_{j}$ or $\mathbf{x}_{j} \leqslant \mathbf{x}_{i}$. Then there exists $a$ sequence $\mathbf{z}$ in $X$ for which $\mathbf{z} \leqslant \mathbf{x}_{i}$ for all $i$.

Proof. For each $n$, choose $\mathbf{y}_{n} \in\left\{\mathbf{x}_{1}, \ldots, \mathbf{x}_{n}\right\}$ so that $\mathbf{y}_{n} \leqslant \mathbf{x}_{1}, \ldots, \mathbf{x}_{n}$. Clearly, $\mathbf{y}_{1} \geqslant \mathbf{y}_{2} \cdots$. For each $n$, let $\mathbf{z}_{n}$ be a subsequence of $\mathbf{y}_{n}$ such that $\mathbf{z}_{n+1}$ is a subsequence of $\mathbf{z}_{n}$ (use induction). If $\mathbf{z}_{n}=\left\{z_{n}^{i}: i \geqslant 1\right\}$, then let $\mathbf{z}=\left\{z_{i}^{i}\right.$ : $i \geqslant 1\}$. Clearly, $\mathbf{z} \leqslant \mathbf{z}_{n} \leqslant \mathbf{y}_{n} \leqslant \mathbf{x}_{n}$ for all $n$.

THEOREM. Let $X$ be a compact topological space of cardinality less than or equal to $\aleph_{1}$. Then $X$ is sequentially compact.

Proof. Let $<$ be a well ordering of $X$ with the property that the set of predecessors of each $x \in X$ is countable.

Now suppose there exists a sequence $\left\{y_{n}\right\}$ in $X$ which has no convergent subsequence. Suppose $x_{1}$ is the smallest element in $(X,<)$; then $\left\{y_{n}\right\} \nrightarrow x_{1}$. Hence, there exists an open set $O_{x_{1}}$ and a subsequence $\left\{y_{n}^{x_{1}}: n \geqslant 1\right\}$ of $\left\{y_{n}\right\}$ such that $x_{1} \in O_{x_{1}}$ and $y_{n}^{x_{1}} \notin O_{x_{1}}$ for all $n \geqslant 1$.

By transfinite induction we shall show that for each $x \in X$, there exists an open set $O_{x}$ and a sequence $\left\{y_{n}^{x}: n \geqslant 1\right\}$ in $X$ for which (1) $x \in O_{x}$, (2) $y_{n}^{x} \notin O_{x}$ for each $n$, and (3) $\left\{y_{n}^{x}\right\} \leqslant\left\{y_{n}^{z}\right\}$ for all $z<x$.

Suppose for all $z<x, O_{z}$ and $\left\{y_{n}^{z}: n \geqslant 1\right\}$ have been defined so that (1), (2),

Received by the editors January 8, 1975.

AMS (MOS) subject classifications (1970). Primary 54D20, 54D30; Secondary 54A25.

() American Mathematical Society 1976 
(3) hold. Now $\{z: z<x\}$ is countable and the family of sequences $\left\{y_{n}^{z}\right.$ : $n \geqslant 1\}, z<x$, satisfies the hypothesis in the Lemma. Hence, by the Lemma, there exists a sequence $\left\{z_{n}: n \geqslant 1\right\}$ for which $\left\{z_{n}\right\} \leqslant\left\{y_{n}^{z}\right\}$ for all $z<x$. But $\left\{z_{n}\right\} \leqslant\left\{y_{n}^{x_{1}}\right\} \leqslant\left\{y_{n}\right\}$ and, hence, $\left\{z_{n}\right\}$ converges to no point. In particular, $\left\{z_{n}\right\} \nrightarrow x$. Thus, there exists an open set $O_{x}$ and a subsequence $\left\{y_{n}^{x}: n \geqslant 1\right\}$ of $\left\{z_{n}\right\}$ such that (1) $x \in O_{x}$, (2) $y_{n}^{x} \notin O_{x}$ for all $n$, and (3) $\left\{y_{n}^{x}\right\} \leqslant\left\{y_{n}^{z}\right\}$ for all $z<x$.

$X=\cup\left\{O_{x}: x \in X\right\}$, and by the compactness of $X$ there exists a finite set $F \subseteq X$ such that $X=\bigcup\left\{O_{f}: f \in F\right\}$. Let $f_{1}<f_{2}<\cdots<f_{k}$ be the elements of $F$. But $\left\{y_{n}^{f_{k}}\right\} \leqslant\left\{y_{n}^{f_{i}}\right\}$ for $i=1, \ldots, k$ and hence $\left\{y_{n}^{f_{k}}\right\}$ is eventually in $e O_{f_{i}}$ for all $i$, $e$ denoting the complement operator. Hence $\left\{y_{n}^{f_{k}}\right\}$ is eventually in $\cap\left\{e O_{f_{i}}: 1 \leqslant i \leqslant k\right\}=\varnothing$, a contradiction.

It is interesting to note that in [2], S. P. Franklin proved that every compact Hausdorff space of cardinality less than $2^{\kappa_{1}}$ is sequentially compact.

The author is grateful to the referee for some helpful suggestions.

\section{REFERENCES}

1. D. Booth, A Boolean view of sequential compactness, Fund. Math. 85(1974), 99-102.

2. S. P. Franklin, On two questions of Moore and Mrowka, Proc. Amer. Math. Soc. 21(1969), 597-599. MR40 \#4923.

Department of Mathematics, Ohio State University, Columbus, Ohio 43210 